\title{
Probing Particle Diffusion around Two Nearby Pulsars with TeV Gamma-Ray Observations from HAWC
}

\author{
Hao Zhou* \\ Los Alamos National Laboratory \\ E-mail: hao@lanl.gov

\section{for the HAWC Collaboration ${ }^{\dagger}$}

Nearby cosmic-ray accelerators, especially pulsar wind nebulae, are possible origins of the local multi-GeV positron excess. Pulsar Geminga and B0656+14, less than $300 \mathrm{pc}$ from the Earth, have been postulated as the main sources of the positron excess. With one and half years of data, the HAWC gamma-ray observatory discovered very extended $\mathrm{TeV}$ gamma-ray structures produced from high-energy electrons and positrons around these two nearby middle-aged pulsars. Morphology studies suggest that the diffusion in the vicinity of these two pulsars is 100 times slower than the average in our Galaxy. This result provides important constraints on the origin of positron excess, but raises questions like why diffusion is so slow at high energies near these pulsars. With more than twice of data from the previous results, we now explore the possibility of energy-dependent diffusion at these sources.

36th International Cosmic Ray Conference -ICRC2019-

July 24th - August 1st, 2019

Madison, WI, U.S.A.

\footnotetext{
* Speaker.

${ }^{\dagger}$ For a complete author list and acknowledgemets, see PoS(ICRC2019)1177 and http://www.hawcobservatory.org/collaboration/icrc2019.php
} 


\section{Introduction}

Pulsar wind nebulae are known as efficient particle accelerators of electrons and positrons. It is suggested that $e^{+} e^{-}$accelerated from pulsars and their surrounding nebulae contribute a large fraction to the leptonic cosmic-ray flux in our Galaxy. One of the most famous anomalies from the cosmic-ray measurements is the "positron excess", i.e. an excess in the locally measured positron flux at energies above $10 \mathrm{GeV}$ relative to the standard predictions of the secondary production in the cosmic rays. This unexpected excess was first discovered by the PAMELA experiment [1], then confirmed with much higher precision by Fermi-LAT [2] and AMS-02 [3]. People have been postulated that these particles could be from a consequence of the annihilation or delay of dark matter, but alternatively, nearby cosmic-ray accelerators, like pulsar wind nebulae, are possible sources as well [4] [5].

Extended "TeV halo" is a newly defined class of $\mathrm{TeV}$ gamma-ray sources [6]. Different from classical pulsar wind nebula, the size of $\mathrm{TeV}$ halo is tens of parsecs, powered by the $e^{+} e^{-}$interacting with the ambient interstellar radiation after they diffuse into the surrounding environment, resulting a very extended gamma-ray structure with unique morphological features. Observations of $\mathrm{TeV}$ halos are important to constrain the efficiency in converting spin-down power to $e^{+} e^{-}$production and the diffusion coefficient in the ambient environment, allowing us to constrain the origin of local positrons.

The $\mathrm{TeV}$ halos around pulsar Geminga and PSR B0656+14 are the first and second identifications of this class. These two nearby mid-aged pulsars have been suggested as two of the best candidates as the origin of high-energy positrons. With one and half years of data, the High Altitude Water Cherenkov (HAWC) observatory detects two very extended gamma-ray structures coincident with the locations of these two pulsars. The spectral and morphological analyses on these two sources suggest that the efficiency on converting spin-down power to $e^{+} e^{-}$production is high, but the diffusion coefficient in the vicinity of the pulsars is 100 times lower than the average in our Galaxy. Assuming the same diffusion coefficient in the environment between these pulsars to the Earth, this result indicates that the leptons emitted from these two sources are unlikely to be the main sources of local positron excess [7]. At $\mathrm{GeV}$ energies, there is recent work that detects a gamma-ray halo around the Geminga pulsar with data taken with Fermi-LAT [9]. This measurement confirms the low diffusion region around Geminga within a distance of about $100 \mathrm{pc}$ from the pulsar.

Two important questions remain: how large the low diffusion region is and how diffusion rate changes with energy. There have been many different theoretical predictions, for example [10] and [11]. In this contribution, we will try to answer these questions with an updated dataset from HAWC and with multi-wavelength studies.

\section{The HAWC Observatory}

The HAWC Observatory, located in central Mexico at $4100 \mathrm{~m}$ above sea level, is sensitive to gamma rays from sub- $\mathrm{TeV}$ to above $100 \mathrm{TeV}$. The HAWC array consists of 300 water Cherenkov detectors covering an area of about 22,000 $\mathrm{m}^{2}$. Four photomultiplier tubes (PMTs) are deployed at the bottom of each tank to detect the Cherenkov light that is produced by air shower particles 
in the water. Thanks to its large field of view of 2 steradians, HAWC has a good sensitivity to extended gamma-ray structures. The full array of HAWC was inaugurated in March of 2015 and has been operational with a duty cycle $>95 \%$. Previous result on Geminga and PSR B0656+14 published by the HAWC collaboration was based on data of about 500 days live-time using the fraction of the hit PMTs in a gamma-ray air shower as a simple energy determination technique. The HAWC collaboration recently developed two energy estimators which allow event-by-event energy reconstruction with improved energy resolution [8]. One of them, the Ground Parameter energy estimator utilizes the fit of measured events to a lateral distribution shape, evaluated at an optimal radius, and corrected for zenith angle. We will report a follow-up study on the TeV halos around Geminga and PSR B0656+14, using the ground parameter technique, with a live-time of about 1000 days of HAWC data.

\section{Results}

The significance maps of the Geminga and PSR B0656+14 region are shown in Figure 1, with different smoothing angles. Extended gamma-ray emission is revealed well coincident with the locations of the two pulsars. The observed radial profiles of Geminga and PSR B0656+14 are shown in Figure 2. The radial profiles are well fitted with a diffusion model, with the diffusion radii consistent with those from the previous publication from HAWC [7].
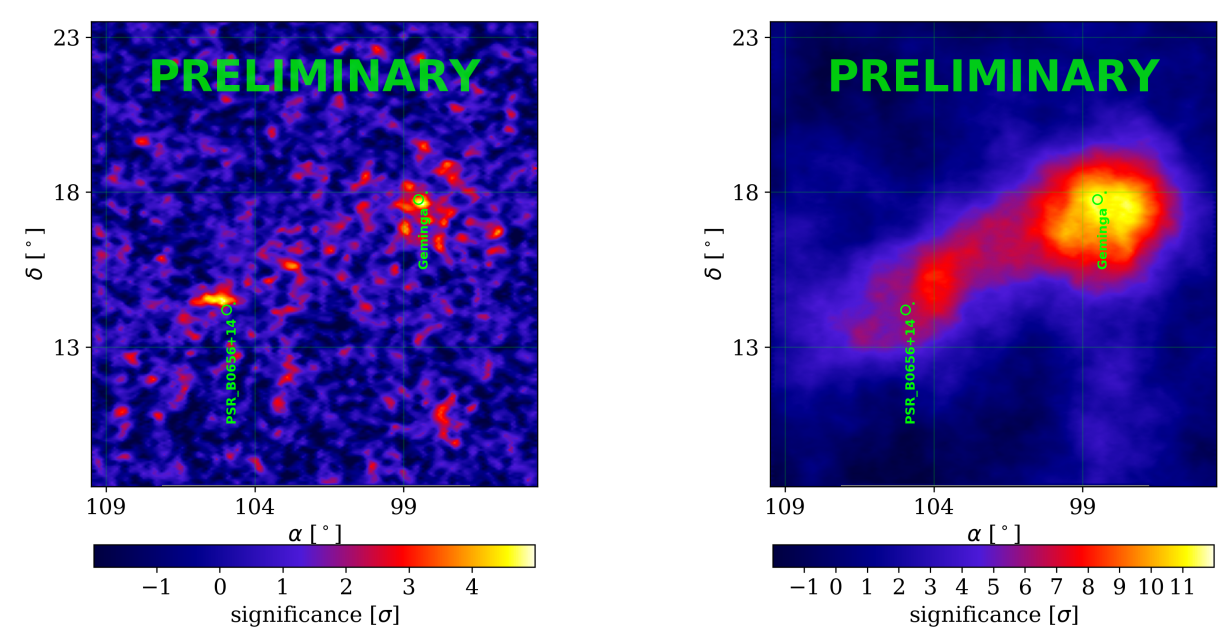

Figure 1: Significance maps of the region of Geminga and PSR B0656+14 in all energy bins with reconstructed energy above $1 \mathrm{TeV}$, smoothed by the point-spread-function (left) and by an additional 2 degree top-hat function (right). Two green markers indicate the positions of pulsar Geminga and B0656+14.

More statistics and better energy resolution allow us to bin the data in energy. Figure 3 6 show the significance maps in energy bins $\mathrm{c}$ to $\mathrm{e}, \mathrm{f}$ to $\mathrm{g}, \mathrm{h}$ to $\mathrm{i}$, and $\mathrm{j}$ to $\mathrm{k}$, corresponding to the reconstructed energy of 1-5.62 TeV, 5.62-17.8 TeV, 17.8-56.2 TeV, and above 56.2 TeV. Both Geminga and PSR B0656+14 are significantly detected in the two mid-energy bins, but not so well 

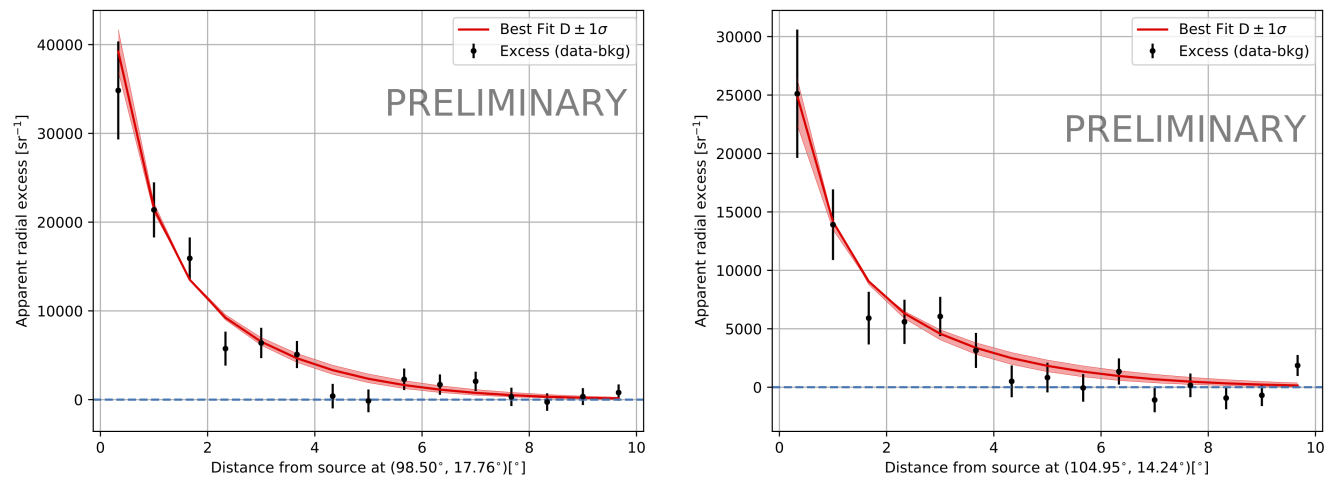

Figure 2: Radial profiles centered on the location of the Geminga pulsar (left) and PSR B0656+14 (right) in all energy bins with reconstructed energy above $1 \mathrm{TeV}$.

in the lowest and highest energy bins. The radial profiles in the two mid-energy bins centered on Geminga are shown in Figure 7.
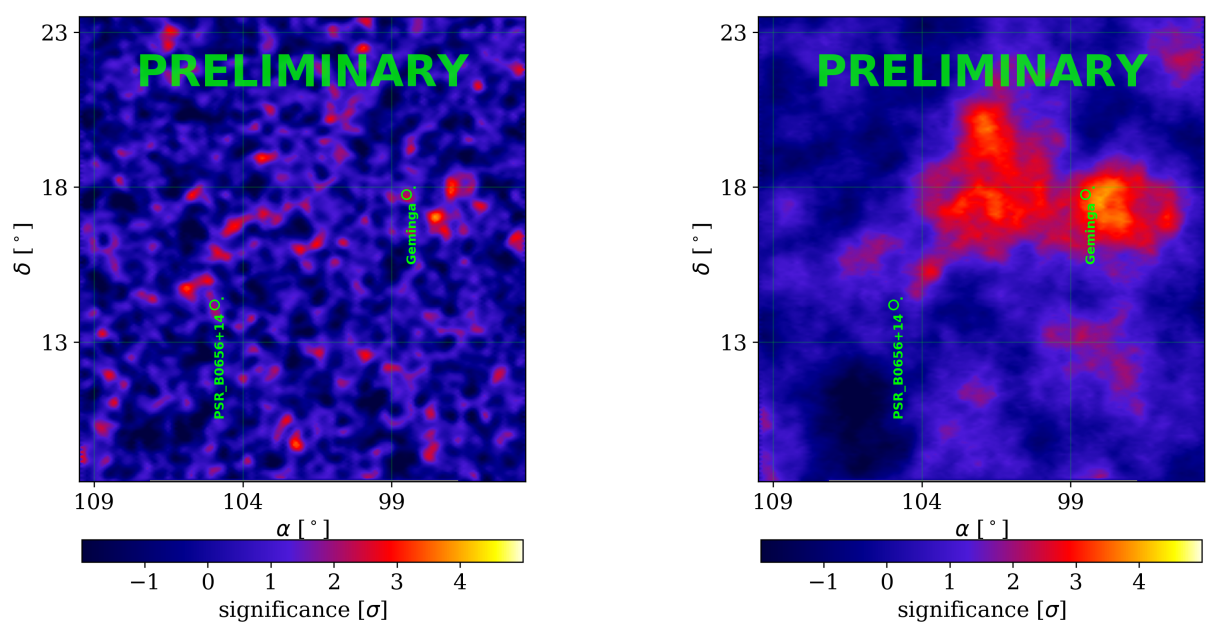

Figure 3: Same as Figure 1 in energy bins c, d, and e with reconstructed energy between 1 and $5.62 \mathrm{TeV}$.

\section{Discussion}

This work has provided experimental results on the TeV halos around Geminga and PSR B0656+14 using over 1000 days of HAWC dataset with better energy estimation technique. The results are consistent with those from the previous HAWC publication. Morphological and spectral studies with HAWC data as well as multi-wavelength studies together with Fermi-LAT data have been carried out and will be presented at the conference. 

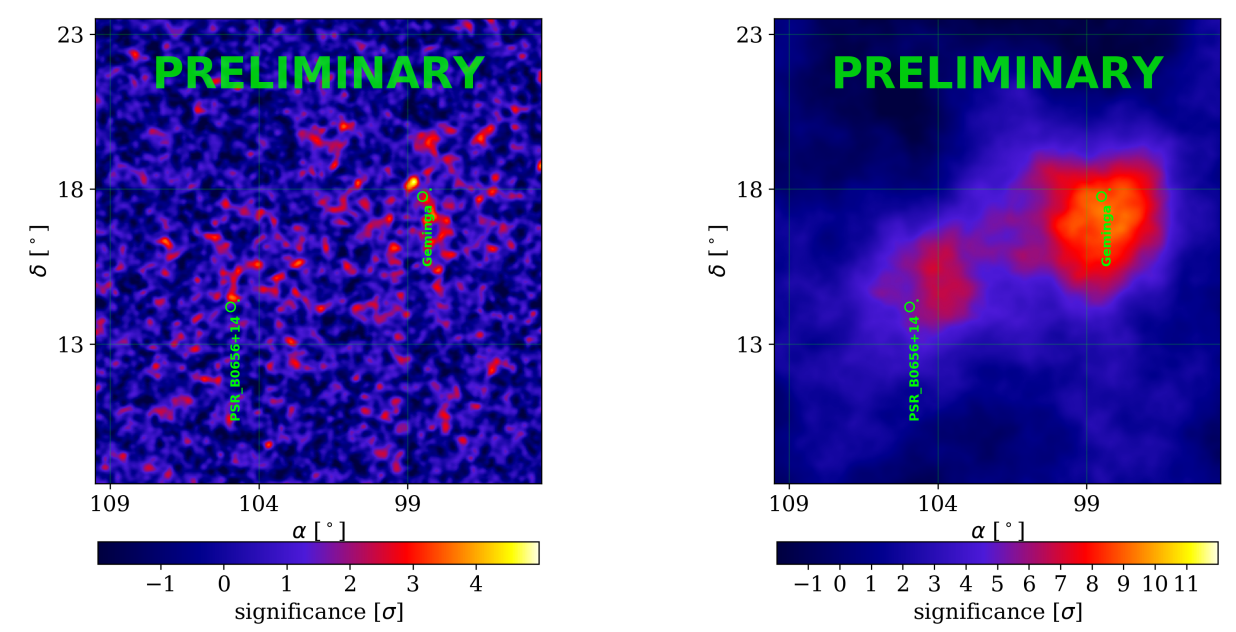

Figure 4: Same as Figure 1 in energy bins $\mathrm{f}$ and $\mathrm{g}$ with reconstructed energy between 5.62 and $17.8 \mathrm{TeV}$.
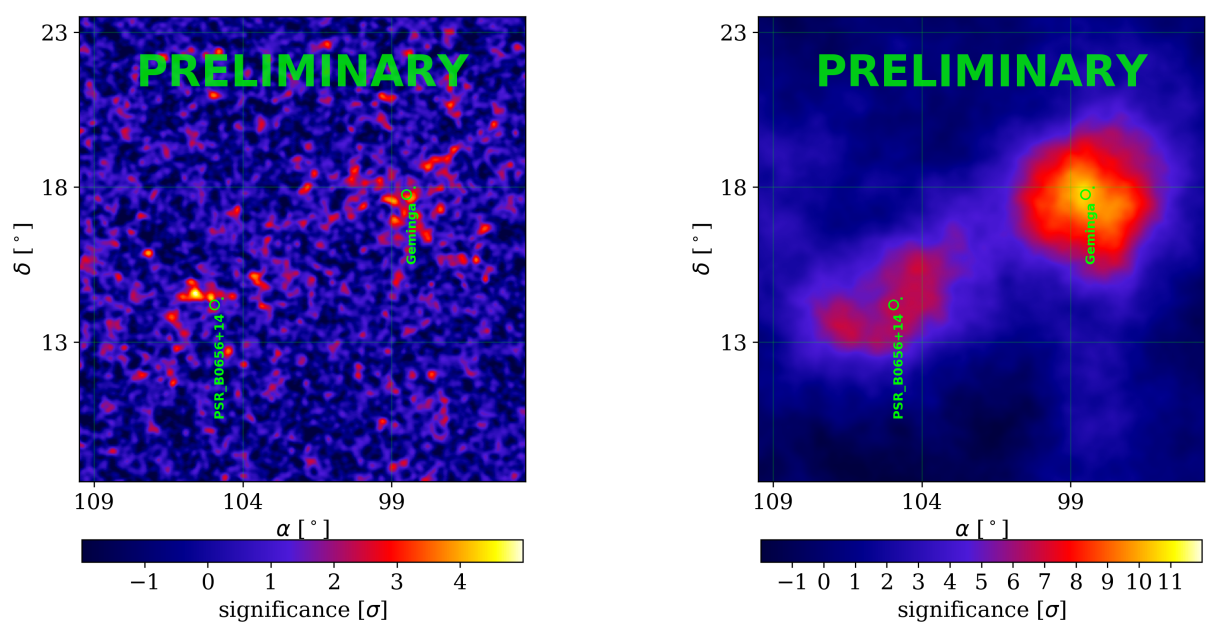

Figure 5: Same as Figure 1 in energy bins $h$ and $i$ with reconstructed energy between 17.8 and $56.2 \mathrm{TeV}$. 

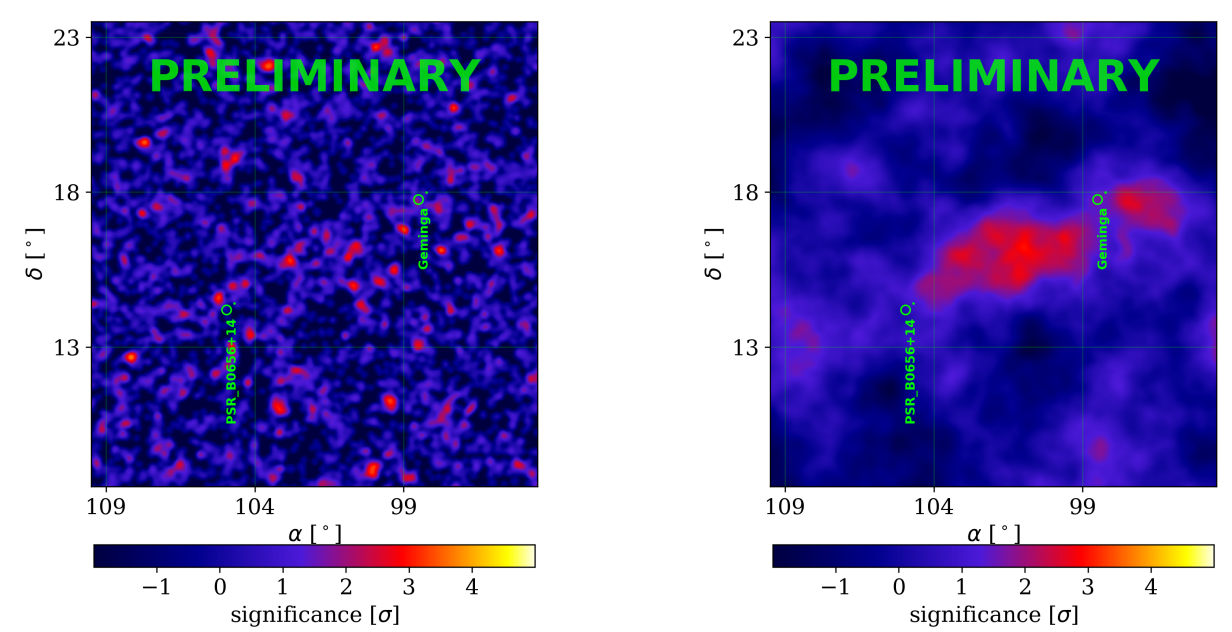

Figure 6: Same as Figure 1 in energy bins j, k, and 1 with reconstructed energy above $56.2 \mathrm{TeV}$.
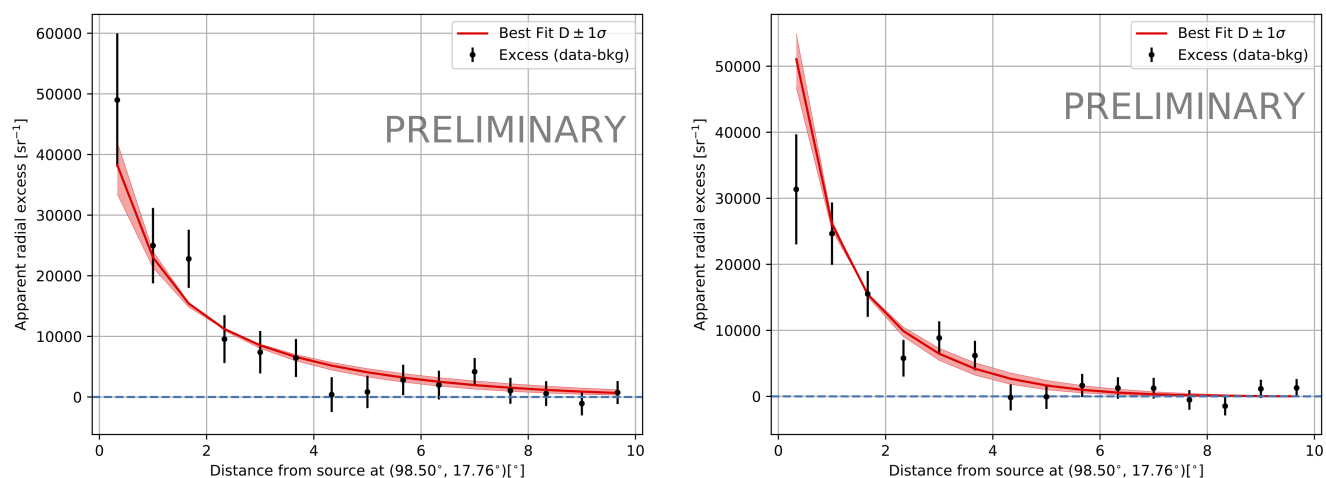

Figure 7: Radial profiles centered on the location of the Geminga pulsar in energy bins f-g (left) and h-i (right).

\section{References}

[1] Adriani et al., Nature 458 (2009) 607

[2] Abdo, A. A. et al., Phys. Rev. Lett. 102 (2009) 181101 [0905.0025]

[3] Aguilar et al., Phys. Rev. Lett. 110 (2013) 141102

[4] Yüksel, H., Kistler, M. D., \& Stanev, T., Phys. Rev. Lett. 103 (2009) 051101 [0810.2784]

[5] Hooper, D., Blasi, P., \& Dario Serpico, P. , Cosmology Astropart. Phys. 1 (2009) 025 [0810.1527]

[6] Linden, T. et al., (2017) [1703.09704]

[7] Abeysekara, A. U. et al. (HAWC), Science 358 (2017) 911 
[8] Abeysekara, A. U. et al. (HAWC), (2019) [1905.12518]

[9] Di Mauro, M. et al., (2019) [1903.05647]

[10] Fang, K., Bi, X. J., \& Yin, P. F., (2019) [1903.06421]

[11] Tang, X. \& Piran, Tsvi, MNRAS 484 (2019) 3 